\title{
Teste de Argolas de Seis Minutos (TA6) em Indivíduos com Disfunção Neurológica
}

\author{
Dannyele Viviane Alecrim ${ }^{1}$, Bianca Bejamim Otoni ${ }^{2}$, Maria Luiza Rodrigues ${ }^{3}$, \\ Dandara Oliveira Souza ${ }^{4}$, Vanessa Pereira Lima ${ }^{5}$, Ana Paula Santos ${ }^{6}$
}

\section{RESUMO}

Os objetivos deste estudo foram obter valores para o teste de argolas de seis minutos (TA6) em uma amostra de indivíduos com disfunção neurológica, comparar estes valores com os valores de referência de adultos saudáveis e verificar a associação do TA6 com o controle de tronco. Estudo transversal realizado com 31 indivíduos com disfunção neurológica de ambos os sexos atendidos em clínica de reabilitação neurofuncional vinculada à universidade federal. Os indivíduos realizaram o TA6 e foram avaliados por intermédio da Escala de Deficiências de Tronco (EDT). Os valores obtidos no TA6 pelos indivíduos com disfunção neurológica foram inferiores aos de adultos saudáveis em todas as faixas etárias estudadas: $30-39(p=0,01 ; T=-$ $5,11) ; 40-49(p=0,05 ; T=-3,06) ; 50-59(p=0,01 ; T=-3,29)$ e 60-69 $(p=0,001 ; T=-6,50)$ anos. Houve comprometimento moderado do tronco. Não houve correlação entre o TA6 e a EDT $(r=0,269 ; p=0,144)$. De acordo com os valores preditos para a população saudável, os pacientes com disfunção neurológica possuem um desempenho funcional e endurance de membros superiores inferiores ao esperado para a sua faixa etária. O TA6 é um teste simples, rápido e barato, útil para reabilitação neurofuncional, pois mimetiza as atividades de vida diária, entretanto não se correlacionou com a EDT.

Palavras-chave: Doenças do sistema nervoso. Atividades cotidianas. Reabilitação neurológica. Tronco.

\section{6-MINUTE PEGBOARD AND RING TEST (6-PBRT) IN SUBJECTS WITH NEUROLOGICAL DYSFUNCTION}

\section{ABSTRACT}

The objectives of this study were to obtain values for the 6-minute pegboard and ring test (6PBRT) in a sample of individuals with neurological dysfunction, to compare these values with healthy adult reference values and to verify the association of 6PBRT with trunk control. Cross-sectional study with 31 individuals of both sexes with neurological dysfunction attended at a neurofunctional rehabilitation clinic linked to the federal university. Individuals performed the 6PBRT and were evaluated through the trunk impairment scale (TIS). The values obtained in 6PBRT by individuals with neurological dysfunction were lower than those of healthy adults in all age groups studied: $30-39(p=0.01 ; T=-5.11) ; 40-49(p=0.05 ; T=-3.06) ; 50-59(p=$ $0.01 ; T=-3.29)$ e $60-69(p=0.001 ; T=-6.50)$ years. There was moderate impairment of the trunk. There was no correlation between 6PBRT and TIS $(r=0.269 ; p=0.144)$. According to predicted values for a healthy population, patients with neurological dysfunction, even in a rehabilitation program, have a lower than expected functional performance and endurance for their age range. The 6PBRT is a simple, fast and inexpensive test, useful for neurofunctional rehabilitation, as it is similar to activities of daily living; however it did not correlate with TIS.

Keywords: Nervous system diseases. Activities of daily living. Neurological rehabilitation. Torso.

RECEBIDO EM: 6/2/2020

MODIFICAÇÕES SOLICITADAS EM: 20/3/2020

ACEITO EM: 18/4/2020

\footnotetext{
Fisioterapeuta graduada pela Universidade Federal dos Vales do Jequitinhonha e Mucuri (UFVJM), Diamantina (MG), Brasil. http://lattes.cnpq.br/ 4240143536588124. https://orcid.org/0000-0002-7038-1123. dannyele_cordeiro@hotmail.com

Graduanda de Fisioterapia pela Universidade Federal dos Vales do Jequitinhonha e Mucuri (UFVJM), Diamantina (MG), Brasil. http://lattes.cnpq. br/2566906701697431. https://orcid.org/0000-0003-4959-5204. bianca-bejamim@hotmail.com

3 Graduanda de Fisioterapia pela Universidade Federal dos Vales do Jequitinhonha e Mucuri (UFVJM), Diamantina (MG), Brasil. http://lattes.cnpq. br/7099622342541633. https://orcid.org/0000-0003-0876-9140. marialuiza_valentim@yahoo.com

${ }^{4}$ Graduanda de Fisioterapia pela Universidade Federal dos Vales do Jequitinhonha e Mucuri (UFVJM), Diamantina (MG), Brasil. http://lattes.cnpq br/0373656061425332.https://orcid.org/0000-0001-5411-3666.dan_dara333@hotmail.com

Fisioterapeuta graduada pela Universidade Federal do Rio Grande do Norte, Mestre em Ciências da Saúde pela Universidade Federal de São Paulo e Doutora em Ciências da Reabilitação pela Universidade Federal de Minas Gerais. Professora do Curso de Fisioterapia e do Programa de Pós-Graduação em Reabilitação e Desempenho Funcional da Universidade Federal dos Vales do Jequitinhonha e Mucuri (UFVJM), Diamantina (MG), Brasil. http://lattes.cnpq. br/5531175017522217.https://orcid.org/0000-0002-0349-3248.vanjaneska@gmail.com

${ }^{6}$ Fisioterapeuta e especialista em Fisioterapia Neurológica pela Universidade Estadual de Londrina; Mestre e Doutora em Neurociências pela Universidade de São Paulo. Professora do Curso de Fisioterapia e do Programa de Pós-Graduação em Reabilitação e Desempenho Funcional da Universidade Federal dos Vales do Jequitinhonha e Mucuri (UFVJM), Diamantina (MG), Brasil. http://lattes.cnpq.br/5486738488474310. https://orcid.org/0000-0003-0952-3249. apsfisio@hotmail.com
} 


\section{INTRODUÇÃO}

Atividades de vida diária (AVDs), como pentear os cabelos, fazer a barba, escovar os dentes, lavar louça, vestir uma roupa ou pegar mantimentos em prateleiras, são comumente realizadas pelos membros superiores (ERAIFEJ et al., 2017; LIMA et al., 2018a). As atividades dos membros superiores requerem estabilização da coluna para que ocorra uma contração efetiva da musculatura do membro superior. Além da atividade muscular aumentada, para a estabilidade postural há aumento do trabalho muscular respiratório nas atividades dos membros superiores (HAN; KIM, 2018; PEETERS; GROOT; GEURTS, 2018).

$O$ teste de argolas de seis minutos (TA6) mimetiza as AVDs (JANAUDIS-FERREIRA et al., 2012). Esse teste é utilizado para avaliar a funcionalidade e endurance de membros superiores e pode ser utilizado na prática clínica por ser um método simples e barato (ZHAN et al., 2006). É um teste que exige coordenação motora, e, do ponto de vista cardiorrespiratório, não é intenso; é um teste sem carga e de curta duração (LIMA et al., 2018a).

Em indivíduos saudáveis o TA6 apresenta relação com o nível de atividade física e idade (LIMA et al., 2018a); em indivíduos com doença pulmonar obstrutiva crônica (DPOC) tem correlação com a capacidade pulmonar total e AVDs de membros superiores (TAKEDA et al., 2013). Não foram encontradas descrições do uso do TA6 em indivíduos com disfunções neurológicas. Por suas características e por ser um teste que mimetiza as AVDs, o TA6 torna-se um teste oportuno para estes indivíduos.

A Escala de Deficiências de Tronco (EDT) é um instrumento que avalia o tronco superior e o inferior, de forma estática e dinâmica, englobando todas as amplitudes de movimento do tronco (CASTELLASSI et al., 2009). O tronco é primordial para a realização das AVDs (KORKMAZ et al., 2018), está comprometido em indivíduos com disfunções neurológicas (HARUYAMA; KAWAKAMI; OTSUKA, 2017; PEETERS; GROOT; GEURTS, 2018) e interfere nas funções física e respiratória (SANTOS et al., 2019; VALENZA et al., 2020). Ademais, o controle de tronco é indispensável durante atividades sentadas, posição do TA6, porque interage com o controle dos membros superiores e da cabeça, fornecendo uma base estável (PEETERS; GROOT; GEURTS, 2018).

Dessa forma, ao considerar a aplicação do TA6 em indivíduos com disfunção neurológica, o controle de tronco deve ser levado em conta. Este estudo teve como objetivo avaliar o TA6 em indivíduos com disfunção neurológica atendidos em um centro de reabilita- ção, comparar os valores obtidos com valores conhecidos para adultos saudáveis e correlacionar o TA6 com o controle de tronco avaliado por meio de instrumento específico e validado para a população brasileira.

\section{MÉTODO}

\section{Amostra}

Trata-se de um estudo transversal descritivo, em uma amostra de conveniência composta por indivíduos adultos de ambos os gêneros, que estavam em tratamento no setor de reabilitação neurofuncional de uma Clínica Escola de Fisioterapia vinculada a uma Universidade Federal. Foi aprovado pelo Comitê de Ética em Pesquisa da Instituição pelo CAAE n. 64173817.8.0000.5108.

Foram selecionados os indivíduos que atenderam os seguintes critérios de inclusão: diagnóstico médico de disfunção do sistema neurológico, idade superior a 18 anos, ser paciente da Clínica Escola de Fisioterapia, ter preservada a cognição e a amplitude de movimento ativas de ombro e mãos, concordar e assinar o Termo de Consentimento Livre e Esclarecido. Foram adotados como critérios de exclusão: pressão arterial igual ou superior a $150 / 100 \mathrm{mmHg}$ no momento da avaliação, diagnóstico de doença respiratória e a desistência em qualquer momento do processo de avaliação.

\section{Procedimentos}

Primeiramente, os pacientes responderam, individualmente e em local reservado, a um questionário semiestruturado de perfil sociodemográfico e características clínicas. O TA6 foi realizado com o paciente sentado em uma cadeira, com os quadris, joelhos e tornozelos em aproximadamente 90 graus e as costas apoiadas, em frente a um suporte vertical de madeira onde situavam-se quatro pinos, sendo dois dispostos na altura dos ombros (nível inferior) e os outros dois $20 \mathrm{~cm}$ acima (nível superior) (Figura 1). Em cada pino inferior foram posicionadas 10 argolas. $O$ paciente foi orientado pelos pesquisadores a mover uma argola de cada vez com ambas as mãos, simultaneamente, do nível inferior para o superior e vice-versa, e, assim, continuamente, posto que o objetivo era que o paciente movesse o maior número de argolas possível em 6 minutos. A pontuação total do teste é a quantidade de argolas movidas durante seis minutos. $\mathrm{O}$ paciente foi autorizado a parar e descansar durante o teste se sentisse dispneia, cansaço ou outro desconforto, e retornar imediatamente assim que se sentisse melhor, mantendo a contagem de tempo pelo cronômetro. Incentivo 
Figura 1 - Teste de argolas de seis minutos (TA6)

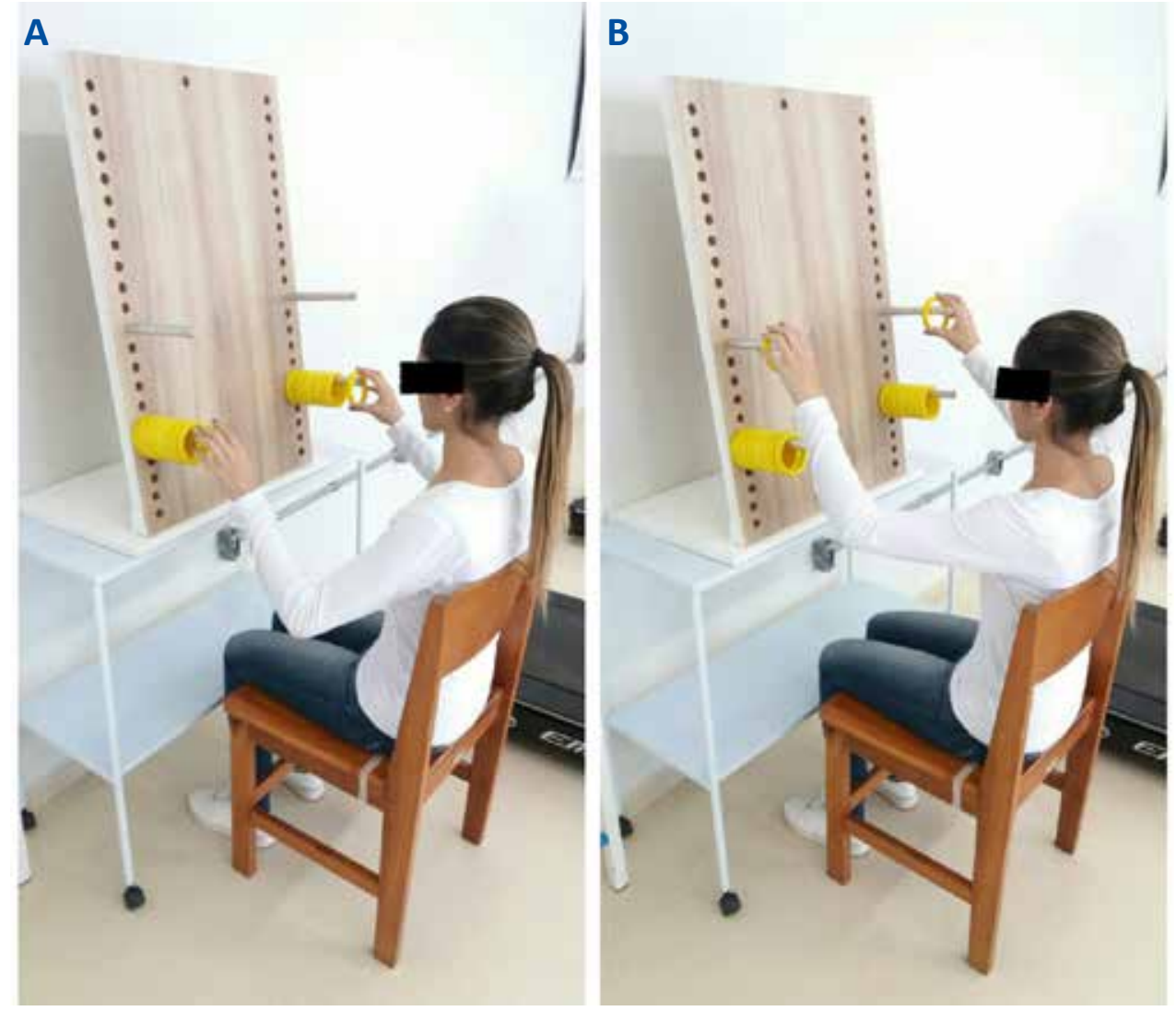

Fonte: Os autores (2020).

padronizado foi dado a cada minuto para o paciente. Antes do teste, os pacientes foram autorizados a mover as argolas para se familiarizarem com o teste (ZHAN et al., 2006; LIMA et al., 2018a). Antes e após a realização do teste foi feita coleta de dados de pressão arterial, frequência cardíaca, frequência respiratória e sensação de dispneia e fadiga de membros superiores pela escala de Borg modificada (zero a dez).

Em A, mostra-se voluntário iniciando a movimentação das argolas dos pinos inferiores para os pinos superiores. Em B, mostra-se o voluntário inserindo as argolas nos pinos superiores.

A seguir foi aplicada a EDT, criada na Bélgica (VERHEYDEN et al., 2004) e validada no Brasil (CASTELLASSI et al., 2009), que avalia o comprometimento do tronco e é constituída por 17 itens, dispostos em 3 subescalas, sendo elas equilíbrio estático, equilíbrio dinâmico e coordenação, avaliados na postura sentada. A pontuação total varia de zero (pior função do tronco) a 23 (melhor função do tronco).

\section{Análise Estatística}

Para a análise estatística foi utilizado o software Minitab 15. Foi realizada uma análise descritiva das variáveis com valores expressos em média, desvio padrão e frequência relativa. Para análise inferencial foi utilizado o Teste Shapiro-Wilk para verificar a normalidade dos dados; o Teste T para uma amostra para comparar os valores do teste de argolas entre adultos saudáveis e indivíduos com disfunção neurológica; o Teste T pareado para comparar os valores de frequência cardíaca, respiratória e sensação de dispneia e cansaço dos membros superiores antes e após o TA6; e o coeficiente de correlação de Pearson para a análise da correlação entre o teste de argolas e o comprometimento do tronco e a idade. As diferenças foram consideradas significativas em $p \leq 0,05$.

\section{RESULTADOS}

Dos 31 pacientes que constituíram a amostra, $55 \%$ eram do sexo feminino. A média de idade foi de 47,4 \pm 15,8 (min: 24 - máx: 69). O valor mínimo de argolas movidas pelos pacientes foi de 64 e o valor máximo foi de 470, com média de 258,2 $\pm 92,1$. Na Tabela 1 estão descritos os valores do TA6 obtidos pelos pacientes neurológicos e pela população saudável (LIMA et al., 2018a) de acordo com cada faixa etária. 
Tabela 1 - Valores do TA6 (número de argolas movidas) obtidos pelos indivíduos estudados com disfunção neurológica, seguido dos valores normativos do

TA6 de acordo com a faixa etária

\begin{tabular}{cccc}
\hline $\begin{array}{c}\text { Faixa } \\
\text { etária }\end{array}$ & $\begin{array}{c}\text { Indivíduos com } \\
\text { DN } \\
\text { Média } \pm \text { DP }\end{array}$ & $\begin{array}{c}\text { Indivíduos } \\
\text { saudáveis* } \\
\text { Média } \pm D P\end{array}$ & p-valor/T-valor \\
\hline $20-29$ & $262,0 \pm 147,1$ & ------------- & ---- \\
$30-39$ & $250,7 \pm 34,9$ & $430,3 \pm 77,1$ & $0,01 /-5,11$ \\
$40-49$ & $194,0 \pm 127,1$ & $414,9 \pm 61,4$ & $0,05 /-3,06$ \\
$50-59$ & $248,0 \pm 85,0$ & $382,7 \pm 59,4$ & $0,01 /-3,29$ \\
$60-69$ & $264,7 \pm 56,6$ & $373,8 \pm 59,4$ & $0,001 /-6,50$ \\
\hline
\end{tabular}

TA6: teste de argolas de seis minutos; DN: disfunção neurológica; DP: desvio padrão da amostra; *: valores normativos referentes ao teste de argolas de seis minutos obtidos pelos indivíduos saudáveis (LIMA et al., 2018a). Teste utilizado: Teste T para uma amostra.

Fonte: Os autores (2020).

Os valores de frequência cárdica e respiratória, Escala de Borg referente à dispneia e fadiga dos membros superiores obtidos após o TA6, foram significativamente maiores que os valores pré-teste e estão descritos na Tabela 2.

Tabela 2 - Valores de frequência cardíaca e respiratória e Escala de Borg referente à dispneia e fadiga de membros superiores antes e após a realização do TA6

\begin{tabular}{cccc}
\hline Variáveis & $\begin{array}{c}\text { Pré-teste } \\
\text { Média } \pm D P\end{array}$ & $\begin{array}{c}\text { Pós-teste } \\
\text { Média } \pm D P\end{array}$ & p-valor \\
\hline FC & $70,8 \pm 8,2$ & $72,8 \pm 8,3$ & $\leq 0,001$ \\
FR & $18,4 \pm 2,4$ & $20,3 \pm 3,1$ & $\leq 0,001$ \\
BR & $0,8 \pm 1,7$ & $2,1 \pm 2,4$ & $\leq 0,001$ \\
BB & $0,8 \pm 1,6$ & $5,1 \pm 2,9$ & $\leq 0,001$ \\
\hline
\end{tabular}

TA6: teste de argolas de seis minutos; DP: desvio padrão da amostra; FC: frequência cardíaca; FR: frequência respiratória; BR: Borg de respiração; BB: Borg de braço. Fonte: Os autores (2020).

Na Tabela 3 estão descritas as pontuações obtidas na escala de tronco, pelos pacientes com disfunção neurológica, com a respectiva média, desvio padrão, valor mínimo e máximo e sua correlação com o TA6. Não houve correlação do TA6 com a idade dos indivíduos com disfunção neurológica $(r=-0,125 p=0,527)$.

Tabela 3 - Pontuação da escala de tronco EDT obtida pelos indivíduos neurológicos estudados e sua correlação com o TA6

\begin{tabular}{|c|c|c|c|}
\hline $\begin{array}{l}\text { Escala de } \\
\text { tronco }\end{array}$ & Média $\pm D P$ & $\begin{array}{l}\text { Mínimo- } \\
\text { Máximo }\end{array}$ & Correlação TA6 \\
\hline EDT & $17,7 \pm 6,5$ & $0-23$ & $r=0,269 \quad p=0,144$ \\
\hline
\end{tabular}

EDT: Escala de Deficiências de Tronco, a pontuação varia de zero (pior função do tronco) a 23 pontos (melhor função do tronco); DP: desvio padrão da amostra; TA6: teste de argolas de seis minutos.

Fonte: Os autores (2020).

\section{DISCUSSÃO}

De acordo com o objetivo proposto neste estudo, foram obtidos valores do TA6 em uma amostra de indivíduos com disfunção neurológica e, a partir desses valores, foi realizada a comparação com valores de referência obtidos por adultos saudáveis de acordo com cada faixa etária (LIMA et al., 2018a), além da correlação entre o TA6 e a EDT, escala validada no Brasil (CASTELLASSI et al., 2009) e utilizada em pacientes adultos com disfunção neurológica (CASTELLASSI et al., 2009; KORKMAZ et al. 2018; HUZMELI et al., 2018; DEMIR; YILDIRIM, 2018; ANABY et al., 2019).

Mesmo sendo consideradas não intensas, do ponto de vista cardiorrespiratório (LIMA et al., 2018a), as variáveis frequência cardíaca e respiratória e a Escala de Borg referente à dispneia e à sensação de fadiga de membros superiores, precisam ser levadas em conta ao realizar o TA6. Neste estudo as variáveis citadas aumentaram significativamente após a realização do TA6, resultado observado também em indivíduos saudáveis (LIMAet al., 2018a; LIMAet al., 2018b).

Foi verificado em indivíduos saudáveis que o teste de exercício não sustentado para membros superiores (unsupported upper limb exercise test - UULEX), teste que, como o TA6, verifica a capacidade funcional dos membros superiores, aumentou o trabalho muscular dos membros superiores, respiratório e da estabilidade postural. O músculo transverso do abdômen apresentou-se ativo progressivamente durante o teste UULEX (JANAUDIS-FERREIRA et al., 2012).

Além de ser associado à idade e nível de atividade física em indivíduos saudáveis (LIMA et al., 2018a), o TA6 correlaciona-se com AVDs de membros superiores em pacientes com DPOC (TAKEDA et al., 2013). Pacientes que apresentam disfunção neurológica possuem, na maioria das vezes, complicações respiratórias, como fraqueza da musculatura respiratória, o que pode ocasionar redução das pressões inspiratória e expiratória máximas e da capacidade pulmonar total, parâmetros estes com forte correlação com TA6 (TAKEDA et al., 2013; VALENZA et al., 2020). Além disso, esses pacientes apresentam limitações na funcionalidade dos membros superiores (KORKMAZ et al., 2018), o que faz com que o TA6 seja um instrumento interessante para a utilização na prática clínica de reabilitação neurofuncional, sendo, ainda, um teste de fácil compreensão e rápida execução (LIMA et al., 2018a).

Podemos perceber, no presente estudo, que os indivíduos com disfunção neurológica apresentaram alteração na função e endurance de membros su- 
periores, em razão do resultado abaixo do esperado para sua idade no TA6, comparado aos valores de referência de adultos saudáveis. Faz-se necessário considerar que a demanda competitiva nos músculos da ventilação para respiração, movimento dos membros superiores e estabilidade da coluna vertebral, podem limitar o desempenho das atividades de levantamento repetitivo (CERNY; UCER, 2004; HAN; KIM, 2018; PEETERS; GROOT; GEURTS, 2018) em indivíduos saudáveis, e é possível que em indivíduos com alguma deficiência neuromuscular ou respiratória o impacto seja maior. Além disso, indivíduos com disfunção neurológica podem apresentar menor resistência e força muscular (SANTOS et al., 2019; VALENZA et al., 2020). Esses dados são importantes, pois os indivíduos avaliados faziam parte de um programa de reabilitação e, mesmo assim, apresentaram resultados bem inferiores aos dos indivíduos saudáveis. Diferentemente de indivíduos saudáveis (LIMA et al., 2018a), a idade dos indivíduos com disfunção neurológica não se correlacionou com o TA6.

Como o TA6 mimetiza as AVDs que utilizam os membros superiores (JANAUDIS-FERREIRA et al., 2012) e essas atividades requerem uma boa estabilidade e mobilidade de tronco (VERHEYDEN et al., 2004; KORKMAZ et al., 2018; SOUZA et al., 2018; SANTOS et al., 2019), a relação entre o TA6 e o controle de tronco era esperada. Percebemos que os indivíduos estudados apresentaram comprometimento moderado de tronco, o que pode causar um impacto negativo na realização das AVDs. O TA6, entretanto, não apresentou correlação com a escala EDT. Vale ressaltar que a EDT foi selecionada para o estudo por ser a escala validada para o Brasil e utilizada por indivíduos com diversas disfunções neurológicas (CASTELLASSI et al., 2009; KORKMAZ et al., 2018; HUZMELI et al., 2018; DEMIR; YILDIRIM, 2018; ANABY et al., 2019). Outras escalas, validadas e utilizadas para um público específico, precisam ser consideradas em estudos próximos. Ademais, a EDT foi escolhida ante a uma posturografia pela forma de execução do TA6 (paciente sentado).

Korkmaz et al. (2018) descreveram uma alta correlação entre a funcionalidade dos membros superiores (habilidade manual na cozinha, em vestir-se, durante a higiene pessoal, durante a execução de tarefas no escritório e outras tarefas de vida diária) e as subescalas equilíbrio dinâmico e coordenação e a pontuação total da EDT $(p \leq 0,005)$. Quanto menor $o$ controle de tronco, pior a função dos membros superiores nos indivíduos com esclerose múltipla remi- tente recorrente, ratificando, assim, a importância do controle de tronco para a funcionalidade dos membros superiores ao realizar AVDs.

O número de pacientes deste estudo poderia ter sido maior, entretanto, para participar do TA6, o paciente tinha de apresentar amplitudes funcionais de ombro, cotovelo e mãos, com capacidade de preensão e soltura das argolas, apresentar PA menor que $150 / 100 \mathrm{mmHg}$, além da cognição preservada; fatores estes comumente encontrados nos indivíduos em reabilitação neurofuncional. Torna-se relevante, contudo, por ser o primeiro estudo, conforme nosso conhecimento, que evidencia valores do TA6 em pacientes com disfunção neurológica e os correlaciona com o controle de tronco. Futuros estudos poderiam relacionar outros fatores, como a força de membros superiores de indivíduos com disfunção neurológica e o TA6.

\section{CONCLUSÕES}

De acordo com os valores preditos do TA6 para a população saudável, os pacientes com disfunção neurológica possuem um desempenho funcional e endurance de membros superiores inferiores do que é o esperado para sua faixa etária. Não houve correlação entre o TA6 e a EDT. O TA6 é um teste simples, rápido e barato, útil para reabilitação neurofuncional, pois mimetiza as AVDs.

\section{REFERÊNCIAS}

ANABY, D. et al. Improving body functions through participation in community activities among young people with physical disabilities. Developmental Medicine \& Child Neurology, v. 31, p. 1-8, 2019.

CASTELLASSI, C. S. et al. Confiabilidade da versão brasileira da escala de deficiências de tronco em hemiparéticos. Fisioterapia em Movimento, v. 22, n. 2, p. 189-199, 2009.

CERNY, F. J.; UCER, C. Arm work interferes with normal ventilation. Applied Ergonomics, v. 35, n. 5, p. 411-415, 2004.

DEMIR, Y. P.; YILDIRIM, S. A. Assessment of Trunk Control in Patients with Neuromuscular Diseases: Validity and Reliability of the Trunk Impairment Scale. Turkish Journal of Neurology, v. 24, p. 130-136, 2018.

ERAIFEJ, J. et al. Effectiveness of upper limb functional electrical stimulation after stroke for the improvement of activities of daily living and motor function: a systematic review and meta-analysis. Systematic Reviews, v. 6, n. 40, p. 1-21, 2017.

HAN, J. W.; KIM, Y. M. Effect of breathing exercises combined with dynamic upper extremity exercises on the pulmonary function of young adults. Journal of Back and Musculoskeletal Rehabilitation, v. 31, n. 2, p. 405-409, 2018. 
HARUYAMA, K.; KAWAKAMI, M.; OTSUKA, T. Effect of Core Stability Training on Trunk Function, Standing Balance, and Mobility in Stroke Patients. Neurorehabilitation Neural Repair, v. 31, n. 3, p. 240-249, 2017.

HUZMELI, E. D. et al. O. Effects of sensory deficits on balance, functional status and trunk control in patients diagnosed with guillain-barré syndrome. Neurosciences Journal, v. 23, n.4, p. 301-307, 2018.

JANAUDIS-FERREIRA, T. et al. How should we measure arm exercise capacity in patients with COPD? A systematic review. Chest, v.142, n. 1, p. 111-120, 2012.

KORKMAZ, N. C. et al. Trunk control: The essence for upper limb functionality in patients with multiple sclerosis. Multiple Sclerosis and Related Disorders, v. 24, p. 101-106, 2018.

LIMA, V. P. et al. Valores de referência para o teste de argolas de seis minutos em adultos saudáveis no Brasil. Jornal Brasileiro de Pneumologia, v. 44, n. 3, p. 190-194, 2018a.

LIMA, V. P. et al. Test-retest reliability of the unsupported upper limb exercise test (UULEX) and six-minute peg board ring test (6PBRT) in healthy adult individuals. Physiotherapy Theory and Practice, v. 34, n. 10, p. 806-812, 2018b.

PEETERS, L. H. C.; DE GROOT, I. J. M.; GEURTS, A. C. H. Trunk involvement in performing upper extremity activities while seated in neurological patients with a flaccid trunk - A review. Gait Posture, v. 62, p. 46-55, 2018.

SANTOS, R. S. D. et al. Relationship between pulmonary function, functional independence, and trunk control in patients with stroke. Arquivos de Neuropsiquiatria, v. 77, n. 6, p. 387-392, 2019.

SOUZA, D. C. B. et al. Controle de tronco, equilíbrio e capacidade de transferência de peso após acidente vascular encefálico: ferramentas de aferição e acompanhamento. Fisioterapia Brasil, v. 19, n. 5, p. 693-699, 2018.

TAKEDA, K. et al. The 6-minute pegboard and ring test is correlated with upper extremity activity of daily living in chronic obstructive pulmonary disease. International Journal of COPD, v. 8, p. 347-351, 2013.

VALENZA, M. C. et al. Respiratory repercussions of neurological diseases and how best to manage them. Expert Review of Respiratory Medicine, v. 14, n. 1, p. 89-102, 2020.

VERHEYDEN, G. et al. The trunk impairment scale: a new tool to measure motor impairment of the trunk after stroke. Clinical Rehabilitation, v. 18, n. 3, p. 326-334, 2004.

ZHAN, S. et al. Development of an unsupported arm exercise test in patients with chronic obstructive pulmonary disease. Journal of Cardiopulmonary Rehabilitation, v. 26, n. 3, p. 180-187, 2006. 